09,05

\title{
Поляриметрия регулярных и стохастических сигналов в магнитооптике
}

\author{
(C) B.C. Запасский
}

Санкт-Петербургский государственный университет, Санкт-Петербург, Россия

E-mail: vzap@rambler.ru

\begin{abstract}
Представлено краткое описание истории применения высокочувствительной лазерной поляриметрии для детектирования малых изменений намагниченности среды - регулярных и стохастических. Описаны принципы и результаты применения спектроскопии спиновых флуктуаций к атомным и полупроводниковым средам. Рассмотрены новые возможности исследований радиоспектроскопических и оптических свойств спиновых систем с использованием методов шумовой спектроскопии. Обсуждены перспективы расширения круга объектов спектроскопии спиновых шумов за счет примесных диэлектриков.
\end{abstract}

DOI: 10.21883/FTT.2019.05.47599.01F

\section{1. Введение}

Среди оптических сред современной фотоники и оптоэлектроники значительное место занимают парамагнетики - материалы и структуры, содержащие элементарные носители момента импульса (спина). Связанные со спиновой подсистемой дополнительные степени свободы проявляются в особенностях взаимодействия оптического излучения со средой, что позволяет применять оптические методы для изучения спиновой динамики. Взаимовлияние оптических и магнитных свойств спиновых систем находит разнообразное применение не только в научных исследованиях, но и для решения прикладных задач обработки и хранения информации [1].

В силу псевдовекторной природы момента импульса (как и вектора намагниченности), тензор диэлектрической проницаемости намагниченной среды также приобретает свойства аксиального вектора, вследствие чего намагниченный парамагнетик становится магнитооптически активным, то есть приобретает способность вращать плоскость поляризации проходящего через него света. Этот замечательный эффект, открытый М. Фарадеем в 1845 г., не только установил прямую связь между двумя областями физики - оптикой и магнетизмом, но и продемонстрировал своеобразие симметрии возмушения среды магнитным полем и, в конечном счете, возможность поляриметрического детектирования намагниченности спиновой системы.

Применительно к современным экспериментальным исследованиям эффект Фарадея часто связывают с вращением плоскости поляризации в любой намагниченной среде, безотносительно к наличию внешнего магнитного поля (которое подразумевается в классическом определении эффекта Фарадея). Намагниченность может быть неравновесной и нестационарной, может быть вызвана действием оптической накачки или высокочастотного магнитного поля и т.д. В любом из этих случаев, намагниченность, направленная вдоль светового луча будет обнаруживаться в эффекте магнитооптического вращения плоскости поляризации.
В настоящей статье мы кратко рассмотрим эволюцию применения эффекта Фарадея в качестве инструмента магнитных измерений, более подробно остановимся на возможностях оптических измерений флуктуаций намагниченности и обсудим условия, при которых оптические измерения флуктуационных характеристик намагниченности среды могут быть эффективными.

\section{2. Методический аспект}

При поляриметрическом детектировании намагниченности магнито-разбавленного парамагнетика важную роль играет чувствительность измерений или, иначе говоря, масштаб минимальных регистрируемых углов поворота плоскости поляризации. Как известно, любые поляриметрические измерения в оптической области спектра основываются на законе Малюса, описывающем преобразование изменений поляризационного состояния света в изменения его интенсивности, после чего задача детектирования изменений поляризации света сводится к задаче чисто фотометрической. Принципиальный предел чувствительности таких измерений накладывается уровнем дробового шума фототока приемника (точнее, уровнем „шума дробового шума“), который, при заданной полосе частот, определяется исключительно величиной этого тока.

Надо заметить, что в долазерные времена вопрос о фундаментальном пределе чувствительности поляриметрических измерений не был актуальным, поскольку чувствительность оптических поляриметров и спектрополяриметров, как правило, определялась уровнем электронных шумов системы регистрации. Впервые вопрос реализации предельной поляриметрической чувствительности был поставлен и решен в работах [2,3], где в качестве источника света использовался лазер. В результате, чувствительность поляриметрических измерений была повышена более чем на 3 порядка величины, и оказалось, что минимальные значения регистрируемых углов (при времени накопления $1 \mathrm{~s}$ и мощности пучка $\sim 1 \mathrm{~mW}$ ) 


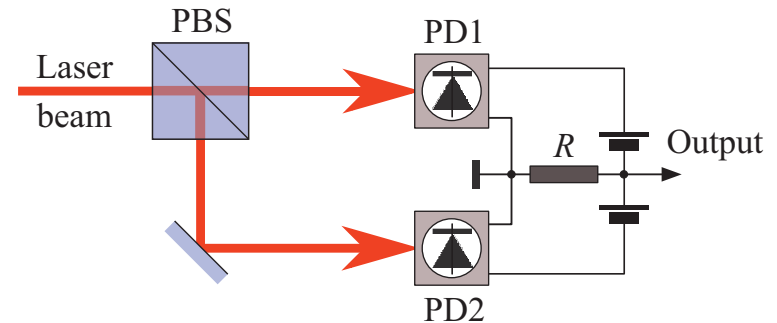

Рис. 1. Балансный детектор - сердце современного лазерного поляриметра. PBS - поляризационный светоделитель, PD1 и $\mathrm{PD} 2$ - фотодетекторы, $R-$ сопротивление нагрузки.

лежат в области $10^{-8} \mathrm{rad}$. Основным препятствием на пути достижения предельной чувствительности были избыточные шумы лазерного источника, для борьбы с которыми был разработан ряд специальных приемов (см. [4]). Наиболее важным элементом схемы подавления избыточных шумов служил балансный детектор (рис. 1), ставший в настоящее время непременным атрибутом поляриметрической схемы. Таким образом, 1976 г. стал годом лазерной революции в поляризационной магнитооптике, применительно к которой высокая поляриметрическая чувствительность означала, в частности, возможность детектирования чрезвычайно малых изменений намагниченности магнито-разбавленных систем.

\section{3. Эфффекты регулярного отклика}

Достигнутый уровень поляриметрической чувствительности придал новый импульс оптическим методам магнитных измерений. Наиболее интересные результаты были достигнуты в работах по оптическому детектированию электронного парамагнитного резонанса (ЭПР) в кристаллах и стеклах с примесными парамагнитными ионами. Было показано, что в большинстве случаев, при лазерно-поляриметрическом детектировании малых изменений намагниченности, отстройка длины волны пробного света от оптического резонанса перестала быть критическим параметром эксперимента: парамагнитный вклад в эффект Фарадея в видимой области спектра способен обеспечить высокую чувствительность безотносительно к особенностям энергетической структуры примесного иона [5].

В результате, диапазон возможностей оптической техники детектирования магнитного резонанса был существенно расширен. В частности, спектр магнитного резонанса двухвалентного иона тулия в кристаллах типа флюорита легко наблюдался в области малых полей и низких частот (порядка ширины линии резонанса) [6], а спектры ЭПР всех (парамагнитных) трехвалентных редкоземельных ионов, которые, как известно, лишены разрешенных оптических переходов в видимой области спектра, надежно регистрировались с использованием одного и того же источника пробного света (гелийнеонового лазера) [7].
Высокая чувствительность лазерных поляриметров была успешно использована также для измерений „нерезонансной“ магнитной восприимчивости примесных парамагнетиков на низких частотах, когда величина оптического отклика зависит от соотношения между частотой приложенного переменного поля и скоростью релаксационного процесса, отвечающего за соответствующий отклик. Такого рода измерения позволяли по частотной зависимости отклика отделить ван-флековский (поляризационный) вклад в магнитную восприимчивость парамагнетика от вклада релаксационного, обнаружить и исследовать процессы межцентровой кросс-релаксации, а также получить достаточно специфичную информацию о характере анизотропии и структуре парамагнитных центров в стеклах $[5,8,9]$.

В наше время, лазерная поляриметрия магнито-оптического отклика с предельно высокой (ограниченной дробовым шумом) чувствительностью измерений заняла важное место в арсенале средств современной оптики, превратившись, практически, в один из ее рутинных методов.

\section{4. Магнитный резонанс в шумах фарадеевского вращения}

Впечатляющая чувствительность лазерной поляриметрии к малым изменениям намагниченности прозрачных парамагнетиков сделала реалистичной идею экспериментального наблюдения флуктуаций равновесной намагниченности среды, связанных с хаотическим движением элементарных магнитных моментов. При измерениях во внешнем магнитном поле возможны две геометрии эксперимента.

В первой из них - так называемая геометрия Фарадея (рис. 2,a), пробный лазерный луч распространяется в исследуемом парамагнетике вдоль внешнего магнитного поля. При этом флуктуации фарадеевского вращения отражают флуктуации продольной намагниченности среды с характерным временным масштабом, определяемым временем продольной релаксации намагниченности $T_{1}$. Спектр такого шума будет описываться лоренцевым контуром, центрированным на нулевой частоте с шириной, соответствующей $T_{1}^{-1}$.

Значительно больший интерес представляет геометрия Фохта (рис. 2, $b$ ), когда пробный световой пучок направлен поперек приложенного магнитного поля. В этом случае свет (регистрирующий вращение плоскости поляризации) откликается на намагниченность, поперечную по отношению к приложенному полю, которая в среднем, очевидно, равна нулю. Однако, любая флуктуационная компонента намагниченности, направленная вдоль луча, в силу прецессионного характера движения всех элементарных носителей момента, будет прецессировать вокруг приложенного поля в течение времени поперечной релаксации $T_{2}$, после чего сменится новой реализацией флуктуационной компоненты с новой амплитудой и новой фазой. Спектр такого шумового сигнала будет 

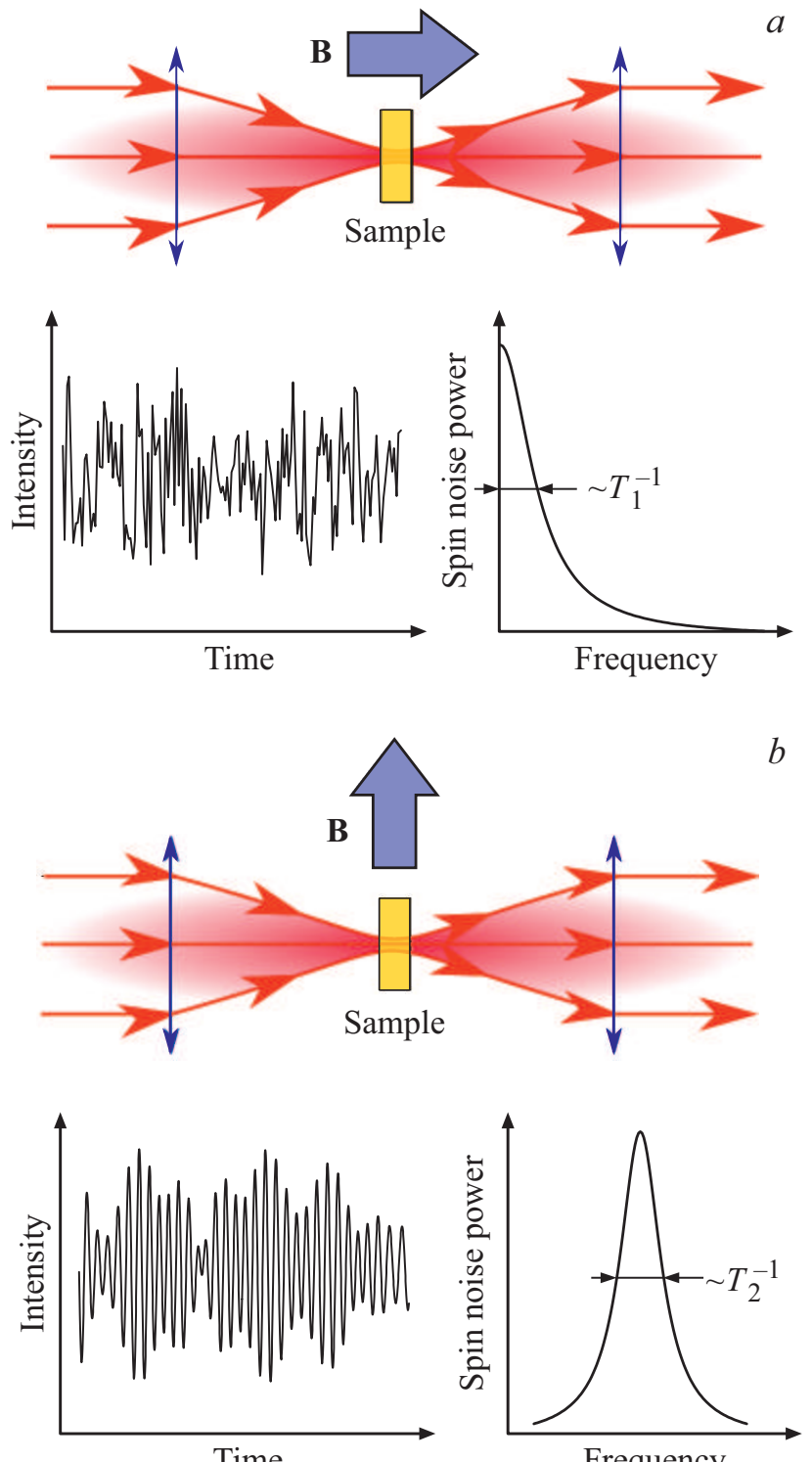

Frequency

Pис. 2. Две базовые геометрии детектирования спинового шума - геометрия Фарадея $(a)$ и геометрия Фохта $(b)$. Ниже каждой оптической схемы представлены условные изображения временной зависимости шума и его спектра.

описываться лоренцевым контуром, центрированным на ларморовой частоте, с полушириной, соответствующей скорости поперечной релаксации намагниченности $T_{2}^{-1}$. Иначе говоря, спектр шума фарадеевского вращения в такой геометрии эксперимента будет представлять собой не что иное, как спектр магнитного резонанса исследуемого парамагнетика.

Эта идея была реализована экспериментально в 1981 г. [10]. В качестве объекта исследований были выбраны пары натрия при температуре $\sim 50^{\circ} \mathrm{C}$. Пробный пучок лазера на красителе настраивался на длину волны, близкую к одной из $D$-линий натрия, где фарадеевское вращение паров испытывало сильный рост, что соответствовало росту эффективности преобразования шумов намагниченности в шумы фарадеевского вращения. Полученные экспериментальные результаты подтвердили правильность выбора объекта исследований - шумовой сигнал магнитного резонанса был зарегистрирован с отношением сигнал/шум порядка $10^{2}$.

\section{5. Особенности шумовой спектроскопии}

Описанный эксперимент по наблюдению магнитного резонанса в спектре шумов фарадеевского вращения с точки зрения академической можно рассматривать, прежде всего, как иллюстрацию к флуктуационно-диссипативной теореме [11], устанавливающей прямую связь между спектром восприимчивости системы и спектром eе спонтанных флуктуаций. Другая сторона эксперимента состояла в том, что он предлагал концептуально новый метод детектирования магнитного резонанса, альтернативный стандартному методу ЭПР Завойского. Новый подход к детектированию магнитного резонанса определял и его отличительные черты, которые сводились, в первую очередь, к следующему:

1. Все стандартные методы регистрации магнитного резонанса подразумевают возбуждение системы высокочастотным магнитным полем. В спектроскопии спиновых шумов пробный лазерный пучок распространяется в среде в области ее прозрачности, не вызывает реальных оптических переходов и, тем самым, не оказывает на среду возмущающего воздействия. В этом смысле спектроскопия спиновых шумов реализует невозмущающий метод магнитного резонанса.

2. Характерной чертой всех известных методов магнитного резонанса является также необходимость магнитной поляризации среды (разницы населенностей магнитных подуровней), чем обеспечивается возможность поглощения высокочастотной энергии. В спектроскопии спиновых шумов для наблюдения сигнала магнитного резонанса магнитной поляризации среды не требуется, что позволяет проводить измерения при высоких температурах и в малых магнитных полях.

3. Несомненное техническое достоинство шумового метода связано с отсутствием канала высокочастотного возбуждения, что радикальным образом упрощало конструкцию спектрометра магнитного резонанса и исключало привязку внешнего поля к частоте возбуждения резонанса.

Отдельного внимания заслуживает вопрос о чувствительности спектроскопии спиновых шумов как метода магнитного резонанса. Величина сигнала спинового шума примерно составляет долю $1 / \sqrt{N}$ от сигнала магнитного насыщения и для макроскопических образцов, очевидно, чрезвычайно мала. Именно по этой причине судьба нового метода ЭПР спектроскопии оказалась непростой: потребовалось почти четверть века для того, чтобы метод спинового шума получил признание в качестве практичного инструмента исследований. Первый 
успешный опыт по наблюдению шумового магнитного резонанса в полупроводниковом образце (n-GaAs) был проведен в 2005 г. [12]. В этом эксперименте время накопления сигнала составило несколько часов. Отмеченное выше отсутствие привязки шумовой спектроскопии к фиксированной частоте высокочастотного индуктора позволило в дальнейшем радикально повысить чувствительность измерений путем использования спектроанализатора с быстрым преобразованием Фурье вместо спектроанализатора сканирующего типа [13-15]. После этого усовершенствования спектроскопия спиновых шумов стала стремительно развиваться - по этой теме опубликованы сотни работ и несколько обзоров (см., напр., [16-19]). Значительную роль в росте популярности этого экспериментального метода сыграл ряд специфических особенностей спектроскопии спиновых шумов, не предсказанных на ранних этапах ее развития и связанных, главным образом, с ее оптическими степенями свободы.

\section{6. Оптические аспекты формирования сигнала спинового шума}

Наличие пробного лазерного пучка с варьируемой длиной волны и варьируемой геометрией предоставляет шумовому методу радиоспектроскопии довольно любопытный канал получения информации. Прежде всего напомним, что величина относительной флуктуации намагниченности, регистрируемой в спектроскопии спиновых шумов, определяется фактором $1 / \sqrt{N}(N-$ число спинов, формирующих сигнал) и растет с уменьшением $N$. Поэтому, в отличие от сигнала регулярного фарадеевского вращения, индуцируемого внешним магнитным полем, величина шумового сигнала зависит от сечения светового пучка, увеличиваясь по мере его уменьшения. По этой же причине, при зондировании объемной среды сфокусированным лазерным пучком, основной вклад в шумовой сигнал вносит область каустики. Это позволяет, перемещая область каустики по объему среды исследовать пространственный рельеф парамагнетика и реализовать таким образом вариант трехмерной магнитной томографии [20].

Отметим, что зависимость величины сигнала от сечения пучка есть не что иное как зависимость сигнала от плотности оптической мощности, что скорее характерно для эффектов нелинейной оптики, подразумевающих нелинейную зависимость оптической восприимчивости среды от напряженности оптического поля. Эта специфика спектроскопии спиновых шумов имеет и ряд других проявлений [21].

Еще одна особенность оптики спиновых шумов, также сближающая ее с оптикой нелинейной, состоит в возможности выявления скрытой структуры оптических переходов и определения характера уширения полос оптического поглощения. В частности, в работе [22] было показано, что характер спектральной зависимости мощности спинового шума от длины волны пробного света (в пределах полосы поглощения оптического перехода) радикальным образом зависит от относительной величины однородного уширения: если контур линии уширен однородно, оптический спектр мощности спинового шума обнаруживает провал в центре линии, если же основной вклад в ширину линии вносит уширение неоднородное, то оптический спектр мощности спинового шума провала не обнаруживает. В промежуточных случаях сопоставимости однородного и неоднородного уширения, по глубине провала можно судить об отношении этих двух вкладов в ширину линии [22]. Как было показано в [23], в более сложных случаях систем с доплеровским механизмом неоднородного уширения, спектр мощности спиновых шумов может „гомогенизироваться“, приобретая провал, типичный для однородноуширенных контуров.

Использование корреляционных свойств шумовых сигналов, формируемых в двухпучковых конфигурациях позволяет реализовать эксперименты типа „зонд-накачка“, обычно неприменимые к эффектам линейной оптики. Эксперименты такого рода базируются, по сути дела, на различии результатов сложения коррелированных и некоррелированных стохастических сигналов. Так в работе [24] применение такого подхода позволило элегантно измерить однородную ширину оптического перехода в ансамбле квантовых точек с огромным неоднородным уширением.

Надо заметить, что, несмотря на кажущуюся простоту формирования сигнала спиновых шумов, в этом вопросе остается ряд неясностей. В работе [25] было показано, что магнитный резонанс в спектре шумов фарадеевского вращения парамагнетика следует интерпретировать как результат гетеродинирования на фотокатоде рассеянной вперед рамановской компоненты пробного света. Такой механизм формирования шумового сигнала подразумевает принципиальную возможность усиления сигнала за счет более полного использования рассеянного света или, по крайней мере, возможность детектирования рассеянной компоненты за пределами апертуры пробного света. Проведенные теоретические исследования [26,27] показали, что апертурные свойства неупругого рассеянного поля могут быть весьма информативны. Надежных экспериментальных данных по этому вопросу, однако, до сих пор не получено.

\section{7. Об объектах спектроскопии спиновых шумов}

Метод спиновых шумов, в принципе, пригоден практически для любых прозрачных парамагнетиков, поскольку все они состоят из конечного числа элементарных магнитов и, в той или иной степени, все они способны обнаруживать эффект Фарадея, связанный с их намагниченностью. Однако, в силу различия эффективности преобразования намагниченности в фарадеевское вращение в разных парамагнетиках, в реальности это не так. 
Как известно, первыми объектами спектроскопии спиновых шумов стали атомы щелочных металлов [10,28,29], высокая магнитооптическая активность которых проявляется вблизи узких линий разрешенных оптических переходов. Следующими объектами спектроскопии спиновых шумов стали легированные полупроводниковые структуры, парамагнетизм которых формируется элементарными носителями спина (электронами, дырками, экситонами) и проявляется наиболее выразительно вблизи края запрещенной зоны полупроводника. Применительно к этим системам метод спектроскопии спиновых шумов оказался чрезвычайно плодотворным. Однако, в настоящее время круг объектов спектроскопии спиновых шумов этими двумя классами сред практически ограничивается.

Между тем, как легко заметить, одни из наиболее популярных объектов ЭПР спектроскопии - кристаллы и стекла с примесными парамагнитными ионами, те самые системы, которые заложили фундамент современной лазерной техники (рубин, неодимовое стекло), пока остаются „за бортом“ нового метода спектроскопии.

Качественно, причины такого состояния дел нетрудно понять. Максимально высокая магнитооптическая активность обычно наблюдается в средах с высокой крутизной дисперсии показателя преломления, т.е., в средах с узкими и мощными особенностями оптического спектра. Это заключение косвенно подтверждается тем фактом, что к длине волны пробного света в спектроскопии спиновых шумов обычно предъявляются достаточно жесткие требования. В этом смысле, кристаллам и стеклам с примесными ионами металлов переходных групп (о которых, в первую очередь, и идет речь) „не повезло“. Разрешенные межконфигурационные переходы этих ионов в кристаллах, как правило, спектрально широкие, а узкие внутриконфигурационные переходы (между уровнями незаполненных электронных оболочек) - запрещены по четности и поэтому являются слабыми. В результате вклады и тех и других переходов в показатель преломления среды на всех длинах волн оказываются не слишком большими.

Масштаб этого различия свойств упомянутых объектов по этому признаку хорошо иллюстрируют оценки, основанные на использовании понятия сечения фарадеевского вращения $\sigma_{\mathrm{F}}[30]$, введенного для оценки эффективности конверсии спиновой поляризации в фарадеевское вращение. Пользуясь результатами работы [31] по измерениям фарадеевского вращения паров ${ }^{87} \mathrm{Rb}$ вблизи оптического резонанса, согласно которым при плотности поляризованных атомов $N \sim 10^{11} \mathrm{~cm}^{-3}$ угол вращения в ячейке длиной $d=2 \mathrm{~cm}$ составляет $\varphi \sim 0.1 \mathrm{rad}$, получаем для сечения фарадеевского вращения $\sigma_{\mathrm{F}}=\varphi / N d \sim 0.5 \cdot 10^{-12} \mathrm{rad} / \mathrm{cm}^{2}$.

В полупроводниковой системе - объемном образце n-GaAs, согласно [30], сечение фарадеевского вращения составляет, приблизительно $10^{-15} \mathrm{rad} / \mathrm{cm}^{2}$.

Для получения характерной величины сечения фарадеевского вращения редкоземельного иона в кристалле мы можем воспользоваться, например, результатами работы [32], согласно которой величина фарадеевского вращения кристалла флюорита (толщиной $2.5 \mathrm{~mm}$ ), содержащего $0.1 \%\left(0.6 \cdot 10^{19} \mathrm{~cm}^{-3}\right)$ двухвалентного тулия (одного из наиболее магнитооптически-активных редкоземельных ионов) составляет $\sim 0.1 \mathrm{rad}$. Учитывая, что указанное значение угла поворота было получено в условиях, близких к магнитному насыщению образца, величина сечения фарадеевского вращения для этой системы составляет $\sim 10^{-19} \mathrm{rad} / \mathrm{cm}^{2}$, что на 4 порядка величины ниже соответствующего значения для полупроводникового кристалла $n$-GaAs.

Приведенные оценки дают представление о масштабе трудностей, которые предстоит преодолеть для надежной регистрации спинового шума в кристаллах с примесными парамагнитными ионами оптическим методом.

\section{8. Заключение}

Шумовой метод детектирования магнитного резонанca, первоначально реализованный на атомных системах, в последнее десятилетие приобрел особую ценность как инструмент изучения свойств полупроводниковых структур. Метод шумовой спектроскопии используется не только для прямых исследований спектров магнитного резонанса и спиновой динамики носителей заряда. Он также предоставляет возможности изучения рельефа плотности носителей, характера уширения оптических переходов, особенностей кинетического движения спинов и пр. Атомы щелочных металлов продолжают оставаться важным объектом спектроскопии спиновых шумов, в частности, играя роль великолепных моделей ансамблей движущихся спинов. Дальнейшее развитие метода шумовой спектроскопии, как нам представляется, будет идти как по пути расширения его возможностей за счет более глубокого понимание закономерностей формирования сигнала спиновых шумов в реальных системах, так и по пути расширения круга объектов этого нового метода.

Работа выполнена при поддержке Российского научного фонда (проект № 17-12-01124).

\section{Список литературы}

[1] A.K. Zvezdin, V.A. Kotov. Modern magnetooptics and magnetooptical materials. CRC Press (1997).

[2] Е.Б. Александров, В.С. Запасский. Оптика и спектроскопия 41, 855 (1976).

[3] R.V. Jones. Proc. Roy. Soc., London, A 349, 423 (1976).

[4] В.C. Запасский. Журн. прикл. спектроскопии 37, 181 (1982).

[5] V.S. Zapasskii. In: Spectroscopy of Solids Containing Rare Earth Ions. Modern Problems in Condensed Matter Sciences. Eds A.A. Kaplyanskii, M.F.Macfarlane. Elsevier, Amsterdam (1987). V. 21, 674 p.

[6] Е.Б. Александров, В.С. Запасский. ФТТ 19, 3083 (1977).

[7] А.А. Антипин, В.С. Запасский. Оптика и спектроскопия 50, 486 (1981). 
[8] Е.Б. Александров, В.С. Запасский. ФТТ 20, 1180 (1978).

[9] В.С. Запасский, Г.Г. Козлов, В.А. Малышев. ФТТ 27, 2742 (1985).

[10] Е.Б.Александров, В.С. Запасский. ЖЭТФ 81, 132 (1981).

[11] Л.Д. Ландау, Е.М. Лифшиц. Статистическая физика. Физматлит, М. (2001). Ч. 1.

[12] M. Oestreich, M. Römer, R.G. Haug, D. Hagele. PRL 95, 216603 (2005).

[13] M. Römer, J.H. Hubner, M. Oestreich. Rev. Sci. Instrum. 78, 103903 (2007).

[14] G. Müller, D. Schuh, J. Hubner, M. Oestreich. Phys. Rev. B 81, 075216 (2010).

[15] S.A. Crooker, J. Brandt, S. Sandfort, A. Greilich, D.R. Yakovlev, D. Reuter, A.D. Wieck, M. Bayer. PRL 104, 036601 (2010).

[16] G.M. Müller, M. Oestreich, M. Römer, J. Hubner. Physica E 43, 569 (2010).

[17] V.S. Zapasskii. Adv. Opt. Photon. 5, 131 (2013).

[18] J. Hübner, F. Berski, R. Dahbashi, M. Oestreich. Phys. Status Solidi B 251, 1824 (2014).

[19] М.М. Глазов. ЖЭТФ 149, 547 (2016).

[20] M. Römer, J. Hubner, M. Oestreich. Appl.Phys. Lett. 94, 112105 (2009).

[21] M.M. Glazov, V.S. Zapasskii. Opt. Express 23, 11713 (2015).

[22] V.S. Zapasskii, A. Greilich, S.A. Crooker, Yan Li, G.G. Kozlov, D.R. Yakovlev, D. Reuter, A.D. Wieck, M. Bayer. Phys. Rev. Lett. 110, 176601 (2013).

[23] M. Yu. Petrov, I.I. Ryzhov, D.S. Smirnov, L.Yu. Belyaev, R.A. Potekhin, M.M. Glazov, V.N. Kulyasov, G.G. Kozlov, E.B. Aleksandrov, V.S. Zapasskii. Phys. Rev. A 97, 032502 (2018).

[24] L. Yang, P. Glasenapp, A. Greilich, D. Reuter, A.D. Wieck, D.R. Yakovlev, M. Bayer, S.A. Crooker. Nature Commun. 5, 4949 (2014).

[25] Б.М. Горбовицкий, В.И. Перель. Оптика и спектроскопия 54, 388 (1983).

[26] G.G. Kozlov, I.I. Ryzhov, V.S. Zapasskii. Phys. Rev. A 95, 043810 (2017).

[27] G.G. Kozlov, I.I. Ryzhov, V.S. Zapasskii. Phys. Rev. A 97, 013848 (2018).

[28] T. Mitsui. Phys. Rev. Lett. 84, 5292 (2000).

[29] S.A. Crooker, D.G. Rickel, A.V. Balatsky, D. Smith. Nature 431, 49 (2004).

[30] R. Giri, S. Cronenberger, M. Vladimirova, D. Scalbert, K.V. Kavokin, M.M. Glazov, M. Nawrocki, A. Lematre, J. Bloch. Phys. Rev. B 85, 195313 (2012).

[31] Zh. Ding, X. Long, J. Yuan, Zh. Fan, H. Luo. Sci. Rep. 6, 32605 (2016).

[32] Y.R. Shen. Phys. Rev. 134, 661 (1964).

Редактор К.В. Емцев 